\title{
Retrieving the size of particles with rough and complex surfaces from two- dimensional scattering patterns
}

\author{
Z. Ulanowski ${ }^{*}$, E. Hirst, P.H. Kaye, R. Greenaway \\ Centre for Atmospheric and Instrumentation Research, Science and Technology Research Institute, University of \\ Hertfordshire, Hatfield, Herts AL10 9AB, United Kingdom. \\ ${ }^{*}$ Corresponding author. Tel.: +441707284604; fax: +441707284185. E-mail address: z.ulanowski@herts.ac.uk
}

\section{ABSTRACT}

Scattered intensity measurement is a commonly used method for determining the size of small particles. However, it requires calibration and is subject to errors due to changes in incident irradiance or detector sensitivity. Analysis of two-dimensional scattering patterns offers an alternative approach. We test morphological image processing operations on patterns from a diverse range of particles with rough surfaces and/or complex structure, including mineral dust, spores, pollen, ice analogues and sphere clusters from 4 to $88 \mu \mathrm{m}$ in size. It is found that the median surface area of intensity peaks is the most robust measure, and it is inversely proportional to particle size. The trend holds well for most particle types, as long as substantial roughness or complexity is present. One important application of this technique is the sizing of atmospheric particles, such as ice crystals.

Keywords: light-scattering; particle; speckle; size; rough; ice

\section{Introduction}

While small particle size is commonly obtained from the magnitude of (integrated) scattered intensity, this technique depends on calibration and is subject to various errors (e.g. due to laser beam power, detector gain, window transmission, background levels etc.). So an alternative procedure that does not depend on absolute intensity measurement may in some cases be preferable, especially in harsh environments like the atmosphere.

In atmospheric science, detailed knowledge of the scattering properties of cloud particles is needed for accurate remote sensing retrievals on the one hand, and for the modelling of radiative impacts of these particles on the other hand. One of the main barriers to achieving these goals is the inability of airborne particle imaging probes to determine the contribution of small ice crystals (that is crystals smaller than about $50 \mu \mathrm{m}$ ) to the total distribution. This is mainly due to the failure of these instruments to resolve the geometric structure of small ice crystals because of the conflicting demands of high optical resolution (high numerical aperture) and large sample volume, as well as the need to avoid particle shattering on probe inlets [1,2]. These difficulties have motivated the development of non-imaging alternatives like the Small Ice Detector (SID) family of instruments, allowing particle characterization using azimuthal or full 2D scattering patterns [3-6].

However, a problem in cloud particle characterization by 2D scattering that has emerged recently is the apparent dominance of ice crystals with rough surfaces or very intricate geometry $[7,8]$. The complexity of these particles and of the resulting patterns, which exhibit speckle-like appearance, makes characterization difficult and in extreme cases prevents particle shape retrieval by direct comparison with theory. Fortunately, 2D patterns can be used to quantify the occurrence of roughness and complexity in atmospheric ice [7], which is one of the determining factors for scattering properties $[9,10]$. Nevertheless, the property that remains to be determined is particle size.

Small particle properties are often retrieved from the dependence of scattering on the polar (scattering) angle. This angular dependence of scattering is a function of the size parameter of the particle and to a lesser degree the refractive index. For single or monodisperse particles this relationship is typically characterized by the presence of oscillations, which phenomenologically can be thought of as interference patterns, and mathematically are a manifestation of the presence of oscillatory functions (e.g. associated Legendre functions for spheres) in the expansions of the scattered field. The larger the size parameter, the higher the frequency of these oscillations - a reflection of the presence of increasing orders in the expansions. Intuitively, therefore, frequency analysis of scattering patterns can be expected to yield particle size information. For example, the cutoff order in 
Gegenbauer polynomial expansions of phase functions is proportional to the size parameter of spheres, although in practice such analysis may require the knowledge of near-complete angular range of the phase function, so that integration required for the transformation from angle to frequency domain can be carried out [11].

The azimuthal dependence of scattering from nonspherical particles can be used to recover information about their size, shape and orientation, although strong a priori assumptions must be made. It has also been shown that some particle information is retained even in the azimuthal frequency spectrum, as demonstrated by the recovery of the size and aspect ratio of prismatic ice crystals $[3,4]$. Additional properties can be expected to be obtainable from two-dimensional (2D) scattering patterns (also called two-dimensional angular optical scattering, TAOS) by virtue of greater information content of the data, although the inversion process is non-trivial $[5,6,12,13]$. One such property is particle roughness, which is associated with the presence of speckle in the 2D patterns, and can be recovered through pattern texture analysis, as more roughness equates with more pronounced speckle [7,9]. The existence of the speckle raises the prospect of particle size recovery too: for example, it was shown in experiments with sphere clusters that the number of intensity peaks in the 2D patterns was proportional to the overall size of the clusters $[13,14]$.

The relationship between the size of rough or complex particles and the properties of the resulting 2D scattering patterns, most notably the numbers and sizes of speckle spots, is the main subject of our investigation. We start by presenting a comparison of two simplified cases, diffraction by a compound aperture and speckle patterns from rough surfaces, and give the results of experimental investigation of scattering patterns from a diverse range of particles.

\section{Theory}

The dependence of speckle size on the size of the scattering object is not surprising, and can be easily illustrated by examining a simplified case, Fraunhofer diffraction on compound apertures consisting of varying numbers and sizes of randomly distributed elements (pinholes). In such a system far-field diffraction pasterns can be obtained using 2D fast Fourier transforms (FFT) of binary arrays see Fig. 1. Examination of the diffraction patterns, which can be thought of as 2D angular spectra, reveals two trends. Firstly, larger overall apertures produce smaller spots (intensity peaks) - fast modulation frequency. Secondly, larger elements produce smaller pattern envelopes (narrower spectra), but with similarly sized spots. This example provides tangible evidence that particle size and roughness information may be recoverable - separately - from forward-scattering $2 \mathrm{D}$ patterns.
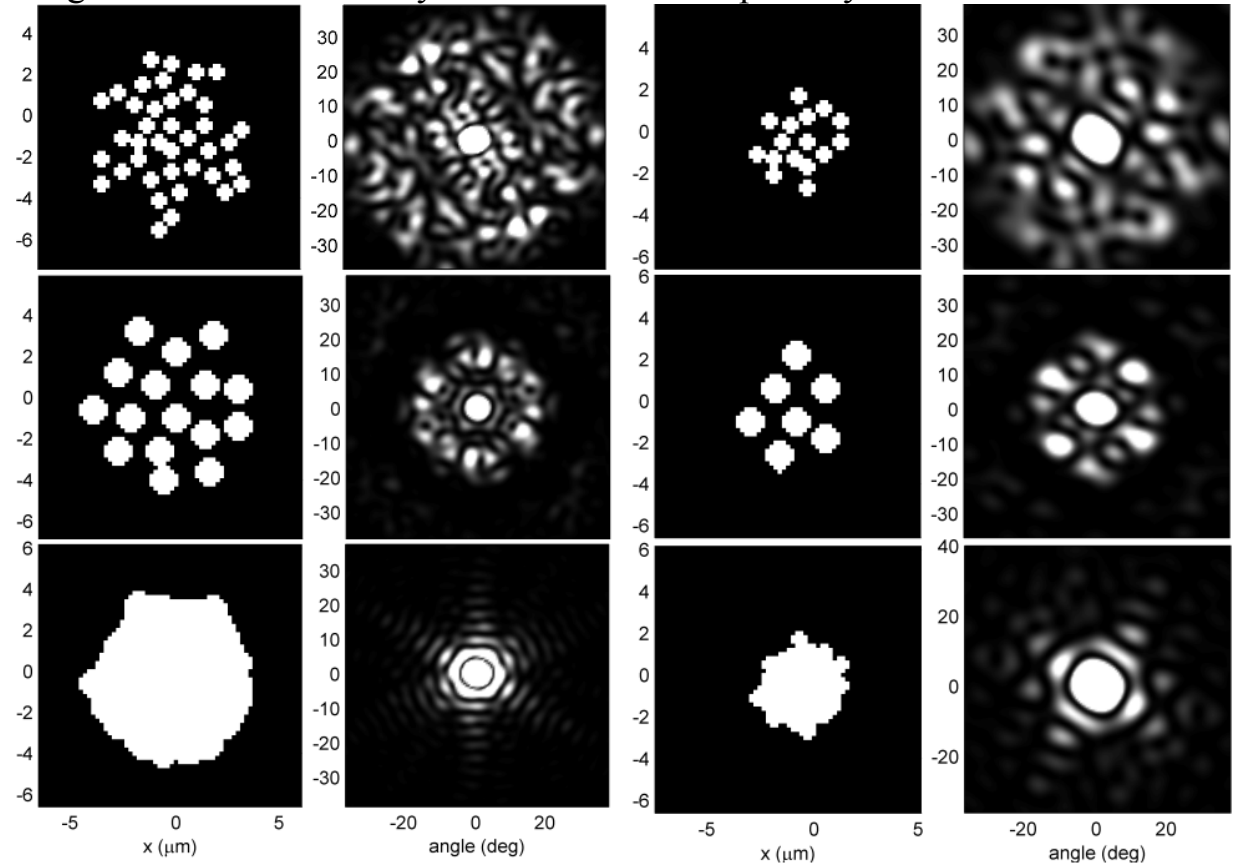

Fig. 1. Compound apertures (columns 1 and 3 ) and corresponding diffraction patterns (columns 2 and 4), computed using fast Fourier transform. A wavelength of $532 \mathrm{~nm}$ was assumed. 
We will now treat the same example analytically. Consider a compound aperture consisting of $N$ small, circular openings, each with radius $r$ and at Cartesian coordinates $\left(x_{m}, y_{m}\right)$ on a flat, opaque screen. The aperture transmittance of the $m$-th aperture can be described as

$$
c_{m}=\left\{\begin{array}{cc}
1 & \left(x-x_{m}\right)^{2}+\left(y-y_{m}\right)^{2} \leq r^{2} \\
0 & \text { otherwise }
\end{array} .\right.
$$

Additionally, for $m=0$ we have $x_{0}=y_{0}=0$. We further assume that the centres of the openings are randomly distributed but confined to a circle of diameter $D$ centred on the origin, i.e. $x_{m}{ }^{2}+y_{m}{ }^{2} \leq D^{2}$. If the openings are non-overlapping, then the entire aperture function becomes

$$
f(x, y)=\sum_{m=1}^{N} c_{m}(x, y) .
$$

In the Fraunhofer approximation the diffracted amplitude due to a plane wave propagating in the direction orthogonal to the aperture can be described as the Fourier transform $\hat{F}$ of the aperture function [15]:

$$
F\left(k_{x}, k_{y}\right)=\hat{F}(f(x, y))=\int_{-\infty}^{\infty} \int f(x, y) e^{i\left(k_{x} x+k_{y} y\right)} d x d y
$$

where the angular spatial frequencies $k_{x}=2 \pi / \lambda \sin \alpha, k_{y}=2 \pi / \lambda^{\sin \beta}, \lambda$ is the wavelength of light and $\alpha, \beta$ are the diffraction angles from the incident direction in the direction of the $x$ and $y$ axes, respectively. For brevity, we have omitted a constant factor in front of the integral in (3). We now denote the diffracted amplitude due to the individual opening at $x=y=0$ as

$$
C_{0}\left(k_{x}, k_{y}\right)=\hat{F}\left(c_{0}(x, y)\right),
$$

which describes the familiar Airy disc with a radius of $1.22 \lambda / 2 r$ radians. From the addition theorem of Fourier transforms the entire diffraction amplitude is

$$
F\left(k_{x}, k_{y}\right)=\sum_{m=1}^{N} C_{m}\left(k_{x}, k_{y}\right)
$$

and from the shifting theorem the diffraction due to the $m$-th opening is

$$
C_{m}\left(k_{x}, k_{y}\right)=C_{0}\left(k_{x}, k_{y}\right) e^{i\left(k_{x} x_{m}+k_{y} y_{m}\right)}
$$

By substituting Eq. (6) into (5) we obtain the final expression for the diffracted amplitude:

$$
F\left(k_{x}, k_{y}\right)=C_{0}\left(k_{x}, k_{y}\right) \sum_{m=1}^{N} e^{i\left(k_{x} x_{m}+k_{y} y_{m}\right)}
$$

Eq. (7) can be interpreted as an Airy disk amplitude modulated by a set of interference fringes with normals in the directions $\left(x_{m}, y_{m}\right)$.

The measured quantity of interest is the intensity of the diffracted wave:

$$
\begin{aligned}
& \left|F\left(k_{x}, k_{y}\right)\right|^{2}=C_{0}\left(k_{x}, k_{y}\right) C_{0}^{*}\left(k_{x}, k_{y}\right) \sum_{m=1}^{N} e^{i\left(k_{x} x_{m}+k_{y} y_{m}\right)} \sum_{n=1}^{N} e^{-i\left(k_{x} x_{n}+k_{y} y_{n}\right)}= \\
& \left|C_{0}\left(k_{x}, k_{y}\right)\right|^{2}\left(N+2 \sum_{m=1}^{N-1} \sum_{n=m+1}^{N} \cos \left(k_{x}\left(x_{m}-x_{n}\right)+k_{y}\left(y_{m}-y_{n}\right)\right)\right),
\end{aligned}
$$

where $\left({ }^{*}\right)$ denotes complex conjugate. Since $x_{m}{ }^{2}+y_{m}{ }^{2} \leq D^{2}$, the interference fringes are characterized by a minimum spacing, or "angular wavelength" $\Lambda_{\min }$,

$$
\Lambda_{\min }=\lambda / D
$$

which occurs when $x_{m}=-x_{n}, y_{m}=-y_{n}$, and $x_{m}{ }^{2}+y_{m}{ }^{2}=D^{2}$, i.e. for a pair of aperture elements at opposite sides of the compound aperture disc.

The case can be easily generalized to aperture elements with non-zero phase delays $\varphi_{m}$, whereby the diffracted amplitude becomes 


$$
F^{\prime}\left(k_{x}, k_{y}\right)=C_{0}\left(k_{x}, k_{y}\right) \sum_{m=1}^{N} e^{i\left(k_{x} x_{m}+k_{y} y_{m}+\varphi_{m}\right)}
$$

and the diffracted intensity

$$
\left|F^{\prime}\left(k_{x}, k_{y}\right)\right|^{2}=\left|C_{0}\left(k_{x}, k_{y}\right)\right|^{2}\left(N+2 \sum_{m=1}^{N-1} \sum_{n=m+1}^{N} \cos \left(k_{x}\left(x_{m}-x_{n}\right)+k_{y}\left(y_{m}-y_{n}\right)+\varphi_{m}-\varphi_{n}\right)\right)
$$

We note that in comparison with Eq. (8) the fringes are merely shifted, without a change to their minimum spacing $\Lambda_{\min }$. Thus the diffraction patterns consist in general of an Airy pattern with radius $1.22 \lambda / 2 r$ modulated by fringes with a minimum angular wavelength of $\lambda / D$ (Eq. 9). Therefore, analysis of measured diffraction patterns could in principle recover separately the fine detail of the aperture structure as represented by the radius $r$, and the entire aperture diameter $D$ - the former from the diffraction pattern "envelope", the latter from the maximum spatial frequency or the resulting speckle spot size.

Indeed, the so called "laser speckle analysis" is a long-established technique for rough surface examination, although it is used for continuous surfaces instead of particles, and most commonly in reflection, rather than transmission mode [16]. The property of interest to us in this context is the size of speckle spots. For "objective speckle", where the scattering is detected directly in the far field, instead of the surface being imaged with a finite-aperture lens, an inverse relationship exists between the speckle spot size and the size of the area on the specimen surface that is illuminated by a (coherent) light beam. The average speckle spot size $D_{s}$ can be expressed in this case approximately as:

$$
D_{s} \approx 4 \lambda z / \pi D
$$

where $\lambda$ is the wavelength of light, $z$ is the distance between the specimen and the detection plane, and $D$ is the diameter of the uniformly illuminated area, assuming that it is a circle. The spot size can be derived from the speckle "coherence area", determined by the normalized autocovariance, in turn obtained from the autocorrelation of the intensity of the speckle pattern [16]. We can presume that a scattering particle can be regarded as being equivalent in this context to the illuminated area on a rough surface, so a similar type of relationship may be expected to exist for discrete particles. We note also that Eqs. (9) and (12) differ only by a constant factor of $4 / \pi$.

These characteristics of the $2 \mathrm{D}$ patterns provide strong evidence that a form of frequency or texture analysis of patterns, especially in the forward scattering region which is dominated by diffraction, may offer ways of retrieving particle size. However, our initial attempts to apply frequency analysis to recover particle size from $2 \mathrm{D}$ scattering patterns were not successful. Such analysis is complicated by the presence of image boundaries, and the beamstop in the case of forward scattering patterns. These features introduce additional frequency components into the resulting spectra. Furthermore, one- or 2D scattering patterns from small particles, while being quasi-periodic, generally do not have constant spatial frequency. For example, the familiar Lorenz-Mie patterns cannot be decomposed directly into simple Fourier spectra; instead, sets of Legendre or Gegenbauer polynomials need to be used as basis functions [11]. Therefore, we focus from here onwards on morphological analysis of the 2D patterns, which does not depend on frequency analysis.

\section{Methods}

The Small Ice Detector model 3 (SID-3) aircraft probe and its laboratory implementations use intensified CCD cameras to capture 2D patterns for a range of scattering angles up to $25^{\circ}$, with the central, low-angle region obscured by a beam stop (rectangular in the aircraft and circular in the lab version used here). Particles are illuminated by a laser beam with elliptical cross-section and a wavelength of $532 \mathrm{~nm}$ [5].

SID-3 2D patterns were obtained from a wide variety of single particles. Rough and smooth mineral dust grains, ice analogue crystals with rough surfaces or complex structure (refractive index 1.31) [9], and silica sphere clusters were deposited on anti-reflection coated glass windows with specified reflectance $<0.2 \%$ per surface. Living cells - fungal spores and single pollen grains - were blown through the detection system in a stream of filtered air, as they were characterized by uniform size. A pollen cluster was attached to a thin glass fibre. Particle size was obtained from optical microscopy images taken at the same orientation as in the scattering data, and expressed as the 
diameter of equal projected area circle. Following the measurements, some particles were also visualized using Scanning Electron Microscopy (SEM). Details of the particles are given in Table 1.

Numbers and surface areas of intensity peaks in the 2D patterns were calculated using image processing and morphology techniques. Data processing was done using MATLAB version 7.4 with the Image Processing Toolbox (The MathWorks Inc.). First, an annular region of interest was selected, to exclude the central beam stop and image margins. At this stage, particle roughness was estimated from the 2D patterns using statistical measures described previously [7], so that smooth particles could be rejected from further analysis. In the next stage, a double-pass median filter was applied to reduce noise and smooth the image; the smoothing was done for several window sizes, so that an optimum could be found, as described below. To obtain a binary image for morphological analysis, the MATLAB functions "imreconstruct" and "imregionalmax" were used, which perform morphological greyscale reconstruction of the image [18], and find regional maxima, respectively. Regional maxima are connected groups of pixels with a constant intensity value, and whose external boundary pixels all have a lower value. The marker image needed for the morphological reconstruction was obtained by multiplying the original image by a constant factor with a value $<1$. For the analysis presented here the factor was selected by trial and error to have a value of 0.6. The surface areas of distinct regions (spots) located in the binary image were calculated using the MATLAB "regionprops" function. The same function also determined spot coordinates, allowing the removal of spots near the boundaries of the region of interest (which may have been truncated). For comparison, a morphological analysis method described in Fernandes et al. [19] was also tested, which uses the extended-maxima function (MATLAB function "imextendedmax") instead of morphological reconstruction followed by finding regional maxima.

Speckle pattern analysis based on morphological processing of images is prone to errors resulting from misidentification of spots. The choice of processing techniques is critical, and the outcome can be sensitive to image brightness, the presence of noise or saturation on the one hand, and parameters such as various thresholds or window sizes on the other hand: different circumstances can lead to very different estimates of the measurands. One frequent artefact is the merging of adjoining intensity peaks in 2D patterns. The effect can be mitigated by adapting the filtration to the size of the speckle and by computing the median, as opposed to mean, average spot area. Furthermore, the processing can be tuned to the specific particle size range.

For these reasons it is helpful to introduce quality criteria indicating the likelihood that the patterns have been correctly processed, especially when automatic procedures not relying on human judgement are used. In the present work the quality measure $Q$ was based on the ratio of the total area $A$ of all spots to the remaining background area $A_{b}$ :

$$
Q=4 C(1-C) \text {, }
$$

where $C=A /\left(A+A_{b}\right)$ can be termed spot coverage. $Q$ is normalized so that it is 0 when either $A$ or $A_{b}$ is zero, and it reaches the maximum value of one when $A=A_{b}$, i.e. when the spots comprise a half of the image area. In practice, $Q$ has typically ranged up to about 0.7 . We have adopted a criterion of $Q>0.4$, below which analysis results are rejected. The threshold chosen on the basis of tests where the correlation coefficient between the true size and the retrieved size was maximized.

We compared two methods for selecting the final solution among those with different values of the quality measure $Q$ : either the solution associated with the smallest smoothing filter window size (while maintaining $Q>0.4$ ), or the solution leading to the largest $Q$. Of the two methods, the former led to solutions more strongly correlated with particle size, and was used to obtain the results reported in the next section.

\section{Results and Discussion}

Scanning electron microscopy (SEM) images of example particles and their corresponding SID-3 2D scattering patterns are shown in Fig. 2, and some of the same patterns converted into binary images in Fig. 3. 

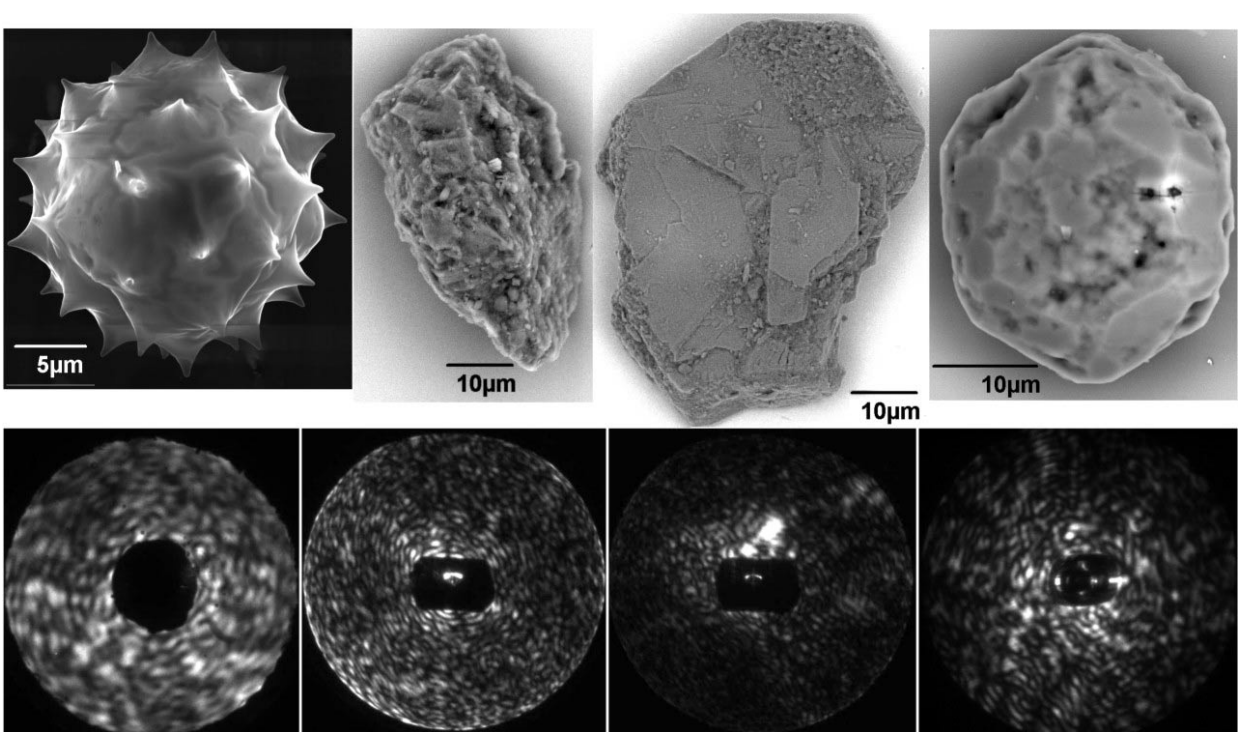

Fig. 2. SEM images (top) and corresponding scattering patterns (bottom) of, from left: Matricaria recutita pollen, rough and moderately smooth mineral dust grains, and a rough ice analogue plate.

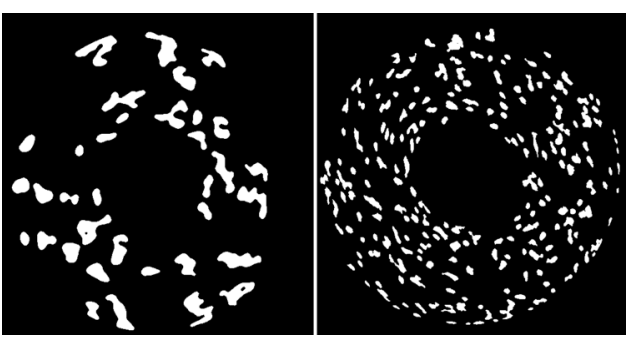

Fig. 3. Scattering patterns from the pollen (left) and the rough mineral dust grain (right) in Fig. 2, converted into binary images. The particles have equivalent sizes of 21 and $37 \mu \mathrm{m}$, respectively.

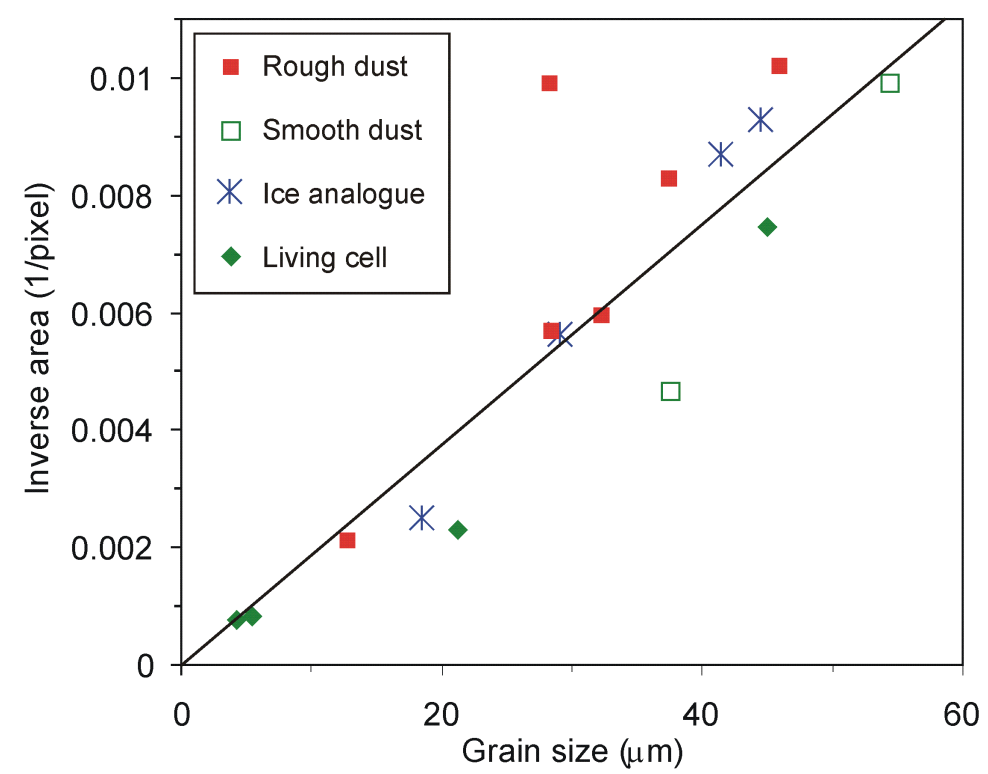

Fig. 4. Inverse of median area of intensity peaks in SID-3 2D scattering patterns from rough mineral dust grains (filled squares), smooth mineral dust grains (open squares), ice analogue crystals (asterisks) and living cells (diamonds), given as a function of particle size expressed as the diameter of equal area circle. The line represents the least squares fit $D=5.3 \cdot 10^{3} / A_{s}$ (see text). 


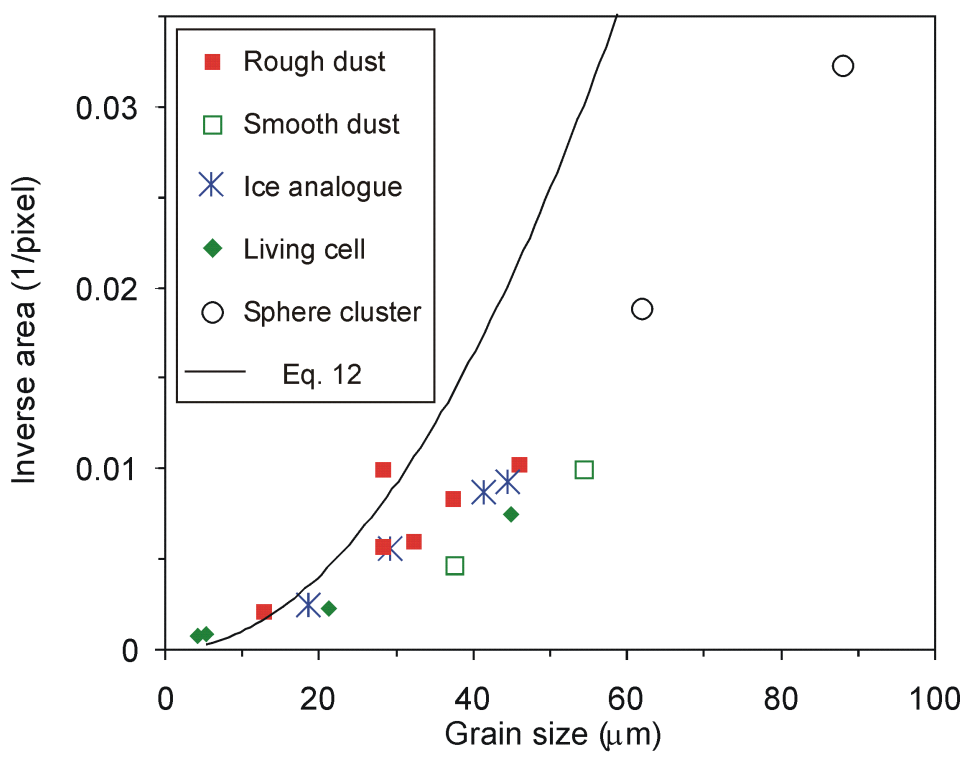

Fig. 5. As Fig. 4 but including sphere clusters (open circles) and the relationship expressed in Eq. (12) (line).

Fig. 4 shows the relationship between particle size and the median surface area of intensity peaks. The area was inversely proportional to the size of the particles, with a correlation coefficient between the size and the inverse of the area equal to 0.89 for the particles shown in Fig. 4, which excludes sphere clusters. The trend was essentially indistinguishable for particles of different origin and refractive index. Only one outlying data point is visible, associated with a $28 \mu \mathrm{m}$ mineral dust grain, and displaying spot area smaller (larger inverse area) than expected from the general trend. This area could have been underestimated if the 2D pattern was not due to a single particle but also included some contamination, making the combined particle larger - a distinct possibility in single particle measurements. This deduction is supported by the fact that a nearly identical particle, with a size of $28.3 \mu \mathrm{m}$, lies close to the trend line, as expected. The particles with moderately smooth surfaces appear to depart from the trend slightly, as the intensity areas were larger than for rough particles of similar size; without these particles the correlation coefficient rose to 0.91 . The least-squares fit to the data in Fig. 4, assuming an intercept of zero and excluding the anomalous particle, corresponded to an expression for particle size $D=5.3 \cdot 10^{3} / A_{s}$, where the median spot area $A_{s}$ is in square pixels and the size is in $\mu \mathrm{m}$, which is valid for the specific detection geometry used here. In more general terms, the median solid angle subtended by a speckle spot can be written as $\Omega_{s}=0.0138 / D$, with the solid angle in steradians and the particle size in micrometers. Both expressions are valid for the wavelength of 532 nm.

The number of intensity peaks, a measure suggested previously for sizing particles [13], was directly proportional to particle size but the corresponding correlation was weaker than for the inverse area, and this measure was found to be less robust. The morphological analysis method described in [19] was also tested, but likewise it was found to be less robust and more sensitive to the choice of parameters.

We also examined limited examples of the dependence of spot area on the size of sphere clusters this data is shown in Fig. 5 together with the data from Fig. 4. While the clusters generated smaller speckle size than the trend for the other particles would indicate, the departure was not very large, 30$40 \%$ of the speckle size. This is despite the very different nature of the particles in question.

In contrast, nearly-smooth prismatic ice analogue particles could not be subjected to an analysis by the technique described here because their 2D scattering patterns had low numbers of identifiable speckle spots and the spot areas had very broad distributions, partly due to very large dynamic range of intensities and resulting noise and saturation. These $2 \mathrm{D}$ patterns can be rejected from the analysis a priori, on the basis of the roughness criteria [7] or by using a threshold value for the quality measure $\mathrm{Q}$, as described in the Methods section. However, the speckle pattern from a complex but smoothfaceted "budding rosette" ("budding Bucky ball") crystal $[1,8]$ followed the general trend closely, and the particle could be sized accurately. These findings suggest that the present sizing method requires 
merely the presence of multiple scattering centres extending over the (simple or compound) particle, rather than any specific form of roughness, so that the scattered waves can overlap and interfere to produce the speckle pattern.

Fig. 5 shows also the speckle size (expressed in terms of inverse speckle area) calculated from Eq. (12), assuming image extent of 580 pixels and scattering angle range of $\pm 25^{\circ}$, which is equivalent to the image distance $z=622$ pixels. It is immediately obvious from Fig. 5 that the experimentally found and predicted relationships give broadly similar values but the overall trends are different. Instead of the inverse speckle-size to particle-size relationship of Eqs. (9) or (12) (exponent of -1) we find an exponent of $\approx-0.57$, i.e. in our case spot area rather than size is approximately inversely proportional to particle size (corresponding in Eq. (9) to the diameter of the compound aperture and in Eq. (12) to the diameter of the illuminated area on a rough surface). As a consequence, larger particles produce speckle spots that are larger than in Eq. (9). The reason for this departure is not certain. It is unlikely that the speckle size was increased by the finite size of the aperture of the measurement system (as in "subjective speckle" [16]), because the angular size of the system aperture was orders of magnitude larger than the angular size of even the largest particle. It is possible, however, that the increased spot sizes are a consequence of a reduction in the coherence of the light wave traversing through the particle. In a simplified picture, the particle can be thought of as consisting of two or more surfaces: the first, backward facing one, and any inhomogeneities within the particle, acting as a diffuser reducing the coherence of the wave incident on the forward facing surface. Since the autocorrelation function of speckle intensity, which determines speckle size in Eq. (12), is the Fourier transform of the intensity distribution of the wave emerging from a scattering surface - a consequence of the van Cittert-Zernike theorem [16] - it follows that a reduction in the coherence width of the wave incident on the forward surface will result in an increase of the final speckle size, as observed here. A similar spot size increase is found for doubly-scattered speckle [20].

Finally, we do expect the method to be sensitive to particle orientation, although we have not tested this assertion experimentally. Our theoretical analysis suggests that it would be so, if the particle projected areas were different in different orientations. However, such sensitivity is characteristic of most particle sizing methods, not only those based on light scattering but also microscopy (imaging). In common with other sizing techniques, an improvement would be achieved by recovering not only the size but also some features of particle shape, such as aspect ratio. But this is beyond the scope of the present study.

\section{Conclusions}

Analysis of 2D scattering patterns offers an alternative approach to sizing small particles that does not depend on absolute intensity measurement, so is essentially calibration-free. We tested possible techniques, with special emphasis on morphological image processing operations, using a diverse range of particles with complex or rough surface structure. We found that the average surface area of intensity peaks (spots) in the 2D patterns was inversely proportional to particle size. This relationship was largely independent of particle type and refractive index, as long as the particle was characterized by substantial roughness or complexity. A slightly weaker correlation existed between particle size and the number of intensity peaks. Crucial steps in the processing of the $2 \mathrm{D}$ patterns were median filtration, followed by morphological image reconstruction, which allowed distinct intensity peaks to be reliably found. This procedure was the most robust among the various approaches that were tried. Nonetheless, we must caution that such analysis remains sensitive to variables such as noise in the data on the one hand, and various parameters used in the processing on the other hand. Therefore further studies would be beneficial. They should include investigation of the robustness and accuracy of the technique present here. Also, researching alternatives to image processing, such as frequency analysis, would be advisable, now that the basic principles have been demonstrated.

An inverse relationship between the object size and speckle size, previously found for extended surface analysis using laser speckle (Eq. 12), and derived in this work for compound apertures (Eq. 9), was obeyed only approximately for the particles investigated here, as the power trends were different. We conjecture that this departure is caused by a reduction in the coherence of the incident wave as it traverses the particle from the rear to the forward-facing surface. 
An important application area of this technique is the sizing of atmospheric particles, such as ice crystals or mineral dust, as both these particle types produce 2D scattering patterns with pronounced speckle. Small atmospheric ice is especially difficult to characterize by other methods.

\section{Acknowledgements}

This work was supported by the UK Natural Environment Research Council APPRAISE program grant NE/E011225/1.

\section{References}

[1] Ulanowski Z, Connolly P, Flynn M, Gallagher M, Clarke AJM, Hesse E. Using ice crystal analogues to validate cloud ice parameter retrievals from the CPI ice spectrometer data. In: Proc.14th Int. Conf. Clouds Precipit. Bologna: ICCP; 2004, p.1175-8.

[2] Connolly PJ, Flynn MJ, Ulanowski Z, Choularton TW, Gallagher MW, Bower KN. Calibration of 2-D imaging probes using calibration beads and ice crystal analogues. J Atm Oceanic Technol 2007;24:1860-79.

[3] Ulanowski Z, Stopford C, Hesse E, Kaye PH, Hirst E, Schnaiter M. Characterization of small ice crystals using frequency analysis of azimuthal scattering patterns. In: Proc 10th Int Conf Electromagnetic \& Light Scatt, Bodrum: 2007, p.225-8.

[4] Stopford C, Ulanowski Z, Hesse E, Kaye PH, Hirst E, Schnaiter M, McCall D. Initial investigation into using Fourier spectra as a means of classifying ice crystal shapes. In: Proc 11th Int Conf Electromagnetic \& Light Scatt, Hatfield: 2008, p.247-50.

[5] Kaye PH, Hirst E, Greenaway RS, Ulanowski Z, Hesse E, DeMott PJ, et al. Classifying atmospheric ice crystals by spatial light scattering. Opt Lett 2008;33:1545-7.

[6] Ulanowski Z, Schnaiter M. UV and visible light scattering and absorption measurements on aerosols in the laboratory. In: Reid JP, Signorell R, editors. Fundamentals and Applications of Aerosol Spectroscopy. Boca Raton: CRC Press; 2011, p.243-68.

[7] Ulanowski Z, Kaye PH, Hirst E, Greenaway RS. Light scattering by ice particles in the Earth's atmosphere and related laboratory measurements, In: Proc 12th Int Conf Electromagnetic \& Light Scatt, Helsinki: 2010, p.294-7.

[8] Um J, McFarquhar GM. Dependence of the single-scattering properties of small ice crystals on idealized shape models. Atmos Chem Phys 2011;11:3159-71.

[9] Ulanowski Z, Hesse E, Kaye PH, Baran AJ. Light scattering by complex ice-analogue crystals. J Quantit Spectr Rad Transf 2006;100:382-92.

[10] Yang P, Kattawar G.W, Hong G, Minnis P, Hu Y.. Uncertainties associated with the surface texture of ice particles in satellite-based retrieval of cirrus. IEEE Trans Geosci Remote Sens 2008;46: 1940-7.

[11] Ludlow IK, Everitt J. Inverse Mie problem. J Opt Soc Am A 2000;17:2229-35.

[12] Pinnick RG, Pan YL, Hill SC, Aptowicz KB, Chang RK. Laser-induced fluorescence spectra and angular elastic scattering patterns of single atmospheric aerosol particles. In: Reid JP, Signorell R, editors. Fundamentals and Applications of Aerosol Spectroscopy. Boca Raton: CRC Press; 2011, p.297-320.

[13] Holler S, Pan Y, Chang RK, Bottiger JR, Hill SC, Hillis DB. Two-dimensional angular optical scattering for the characterization of airborne microparticles. Opt Lett 1998;23:1489-91.

[14] Holler S, Zomer S, Crosta GF, Pan YL, Chang RK, Bottiger JR. Multivariate analysis and classification of two-dimensional angular optical scattering patterns from aggregates. Appl Opt 2004;43:6198-206.

[15] Hecht E. Optics. 3rd ed. Reading, Mass.: Addison-Wesley; 1998.

[16] Goodman JW. Speckle phenomena in optics: theory and applications. Greenwood Village: Roberts; 2007.

[17] Ulanowski Z, Ludlow IK. Water distribution, size and wall thickness in Lycoperdon pyriforme spores. Mycol Res 1989;93:28-32.

[18] Vincent L. Morphological grayscale reconstruction in image analysis: applications and efficient algorithms. IEEE Trans Image Proc 1993;2:176-201. 
[19] Fernandes GE, Pan YL, Chang RK, Aptowicz K, Pinnick RG. Simultaneous forward- and backward-hemisphere elastic-light-scattering patterns of respirable-size aerosols. Opt Lett 2006;31:3034-6.

[20] Yoshimura T, Kato K, Nakagawa K. Surface-roughness dependence of the intensity correlation function under speckle-pattern illumination. J Opt Soc Am A 1990;7:2254-9.

Table 1. Details of investigated particles.

\begin{tabular}{lccc}
\hline \multicolumn{1}{c}{ Particle } & Size $(\mu \mathrm{m})^{\text {a }}$ & Refractive index & Origin \\
\hline Mineral dust & $13,37,28,28,32$, & $\sim 1.5-1.6$ & various \\
& $38,46,54$ & & \\
Pollen, Matricaria recutita $^{\mathrm{b}}$ & 21 & & UK \\
Pollen cluster, Bellis perennis $^{\mathrm{b}}$ & 45 & & UK \\
Spore, Handkea excipuliformis $^{\mathrm{c}}$ & 5.4 & $\sim 1.49$ (wall) & UK [17] \\
Spore, Calvatia gigantea $^{\mathrm{c}}$ & 4.2 & $\sim 1.49$ (wall) & UK [17] \\
Ice analogue crystals $^{\mathrm{d}}$ & $18.5,29,41,44$ & 1.31 & {$[9]$} \\
Silica sphere cluster, 2 spheres & 62 & 1.46 & \\
Silica sphere cluster, 5 spheres & 88 & 1.46 & \\
\hline
\end{tabular}

${ }^{\mathrm{a}}$ Diameter of equal projected area circle.

${ }^{\mathrm{b}}$ Family Compositae.

${ }^{\mathrm{c}}$ Fungal group Gasteromycetes (puffballs).

${ }^{\mathrm{d}}$ Three rough crystals and one complex "budding rosette" (size $44 \mu \mathrm{m}[1,8]$ ). 